Proceedings

\title{
Influence of Sensor Network Sampling Rate on Multivariate Statistical Condition Monitoring of Industrial Machines and Processes ${ }^{\dagger}$
}

\author{
Tizian Schneider ${ }^{1,2, *}$, Nikolai Helwig ${ }^{1}$, Steffen Klein ${ }^{1,2}$ and Andreas Schütze ${ }^{1}$ \\ 1 Lab for Measurement Technology, Department Systems Engineering, Saarland University, \\ 66123 Saarbruecken, Germany; n.helwig@lmt.uni-saarland.de (N.H.); s.klein@lmt.uni-saarland.de (S.K.); \\ schuetze@lmt.uni-saarland.de (A.S.) \\ 2 ZeMA-Centre for Mechatronics and Automation Technology, Department Sensors and Actuators, \\ 66121 Saarbruecken, Germany \\ * Correspondence: t.schneider@zema.de; Tel.: +49-681-85787-48 \\ + Presented at the Eurosensors 2018 Conference, Graz, Austria, 9-12 September 2018.
}

Published: 26 December 2018

\begin{abstract}
In this paper, the deterioration of statistical fault classification of a hydraulic system and an electromechanical cylinder EMC due to reduced sampling rates of sensor nets is shown. As a result, two types of faults can be distinguished: On the one hand, degradation processes which primarily show static symptoms over the whole working cycle and, thus, are less susceptible to reduced time resolution; on the other hand, the detection of faults with symptoms localized in time, e.g., during transients, is significantly degraded. Furthermore, the EMC example shows the importance of data representation that needs to be adapted to the sampling rate.
\end{abstract}

Keywords: machine learning; sampling rate; condition monitoring; fault classification; remaining useful lifetime estimation; data fusion

\section{Introduction}

The paradigm of condition based maintenance in industrial applications requires a multitude of sensor signals. With rising demands and increasing complexity of the systems, the number of installed sensors and their sampling rate are constantly growing. Thus, the data rate to be analyzed and stored has to be scrutinized in the context of automated statistical analysis (machine learning) for condition monitoring. Here, many parameters of instrumentation, data acquisition and the signal processing chain affect the classification performance. In this paper, the impact of reduced sampling rates of sensor nets on the fault classification of a hydraulic system [1] (Figure 1a) and the remaining useful lifetime estimation of an electromechanical cylinder [2] (Figure 1b) is addressed. 

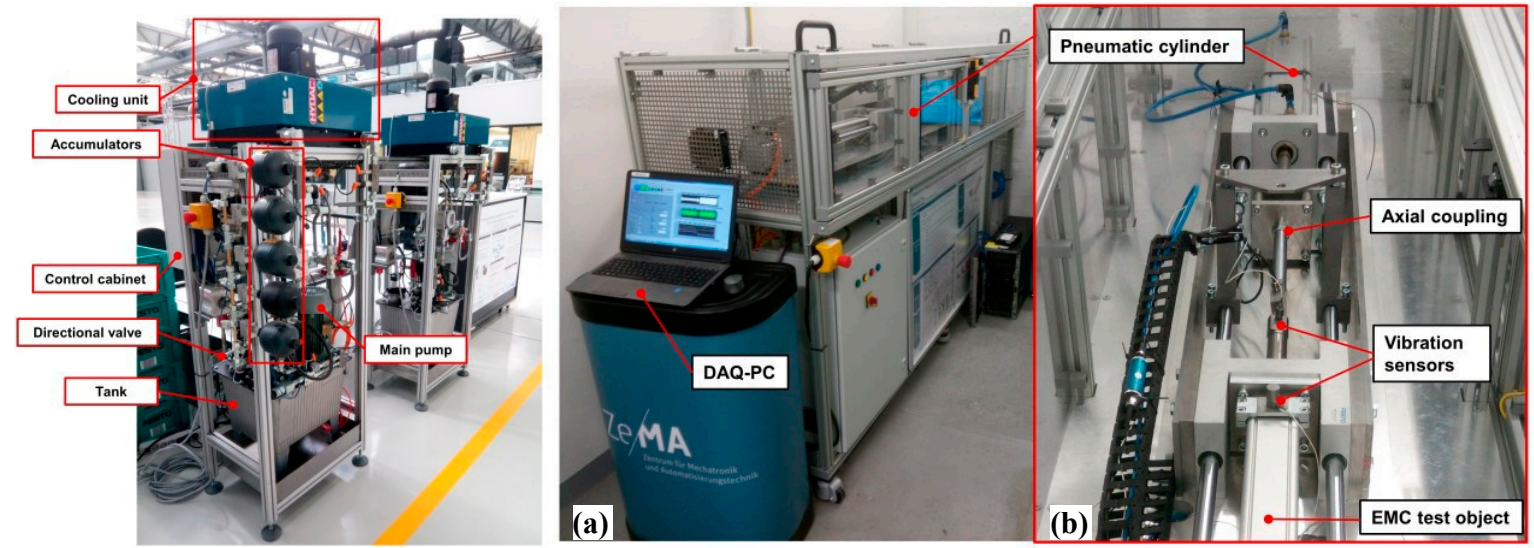

Figure 1. Experimental setups used in this study: (a) hydraulic system equipped with 17 process sensors with experimentally generated fault states of the components cooler, directional valve, pump and accumulator, (b) test bench for residual lifetime analysis of electromechanical cylinders (EMC) based on vibration, sound emission and motor current analysis.

\section{Materials and Methods}

For the hydraulic system the analysis of 17 process sensors (pressures, flows, temperatures, vibration) is performed to identify experimentally generated fault scenarios during a constant and randomized working cycle. These fault scenarios comprise the variation of fault conditions of the components cooler, valve, pump and accumulator. To study the effect of reduced sampling rates of sensor signals the original data collected with a maximum of $100 \mathrm{~Hz}$ are artificially reduced by a factor 2, 5, 10, 20 and 50, respectively. For data analysis statistical and signal shape features are extracted from all sensor signals. For each fault scenario the best features are selected by Spearman correlation between the individual feature and the fault severity. Further dimensionality reduction is performed by applying Linear Discriminant Analysis [3]. Classification of fault severity is then performed by Mahalanobis distance classification.

For the EMC the results of an operational lifetime experiment are shown. During the experiment the EMC is repeatedly driven at maximum speed against a pneumatic load that applies a constant pulling force matching the maximum load of the EMC to simulate quick wear down. Until EMC failure a total of 612,000 cycles (every 100th is used for data analysis) are recorded by three electrical motor current sensors (1 MHz sampling rate), three accelerometers, one microphone (100 $\mathrm{kHz}$ sampling rate each) and eight process sensors (e.g., axial force and position; $10 \mathrm{kHz}$ sampling rate each). Data analysis was performed by a fully automatic approach that automatically selects the best out of five possible feature representations (each capturing information from a different domain) based on cross-validation of feature selection by highest Pearson correlation to lifetime, followed again by LDA and Mahalanobis classification [4]. For the LDA the EMC lifetime is split into 20 equidistant segments each representing $5 \%$ of the lifetime. Without downsampling features are best extracted from frequency domain and most of the information is contained in the frequency spectrum $<1 \mathrm{kHz}$. Therefore, the sampling rate was artificially reduced to $2 \mathrm{kHz}, 1 \mathrm{kHz}, 500 \mathrm{~Hz}, 100$ $\mathrm{Hz}$ and $50 \mathrm{~Hz}$ using downsampling with an antialiasing low passfilter.

\section{Results}

Figure 2 shows the impact of the sampling rate on the fault grade classification accuracy of the hydraulic machine. Fault scenarios with primarily static symptoms (cooling efficiency degradation, pump leakage) are not affected by reduced sampling rates whereas the reduced time resolution impairs the classification rates for monitoring of valve switching degradation and accumulator pre-charge pressure which are based on short-time features during signal transients. Especially without antialiasing low pass filters the accuracy degrades significantly $(-15 \%$ and $-13 \%$ for the 
accumulator and valve, respectively). As a result, two types of faults have to be distinguished: On the one hand, degradation processes which primarily show static symptoms over the whole working cycle and, thus, are less susceptible to reduced time resolution; on the other hand, the detection of faults with symptoms localized in time, e.g., during transients, which are significantly degraded. Figure 3 shows that evaluation of more than 20 features attenuates the information loss due to reduced time resolution which is especially severe if only a single feature is used for classification.

Figure 4 shows the results for the remaining useful lifetime estimation of the EMC. As seen in the left part of the figure, the predictive performance of features extracted from the frequency domain drops significantly $(-14 \%)$ when the sampling rate is reduced from 500 to $100 \mathrm{~Hz}$. This can be explained by the loss of important signal characteristics like motor synchronized vibrations in the spectrum between $50 \mathrm{~Hz}$ and $250 \mathrm{~Hz}$ that cannot be captured at $100 \mathrm{~Hz}$ due to the Nyquist criterion. These belong to the highest ranked features for high sampling rates. This is also shown in Table 1, which shows the best classification rate achieved for the tested sampling rates. As expected, the reduced sampling rate limits the amount of signal characteristics that can be used to model the remaining useful lifetime and therefore deteriorates classification performance. Although this is independent of feature representation the representation needs to be adapted to the sampling rate, i.e., instead of Fourier coefficients the best performance at low sampling rates is achieved by PCA.

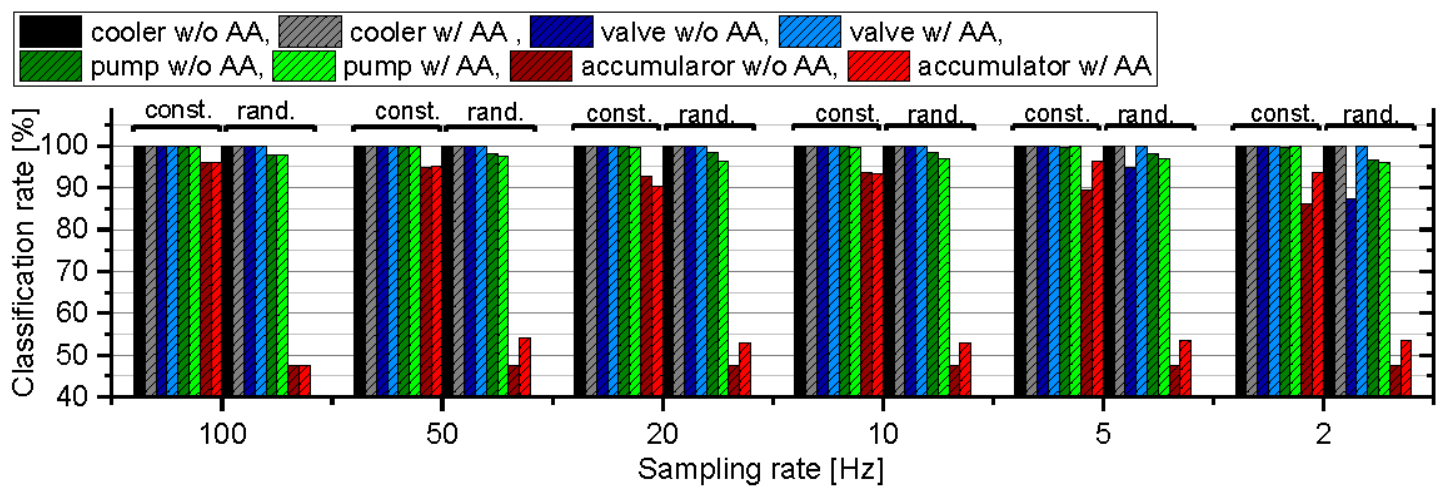

Figure 2. Classification rates for individual component faults depending on the sampling rate for the hydraulic test bed with constant working cycles (const.) and randomized processes (rand.). Direct sample rate reduction using only every $x$-th data point (w/o AA) and downsampling with preceding FIR antialiasing lowpass filter (w/AA) are tested separately and compared. The prediction perfor-mance of the 20 features with highest correlation to the fault state (Spearman correlation feature selection) were evaluated with 10-fold cross-validation based on Mahalanobis distance classifier.
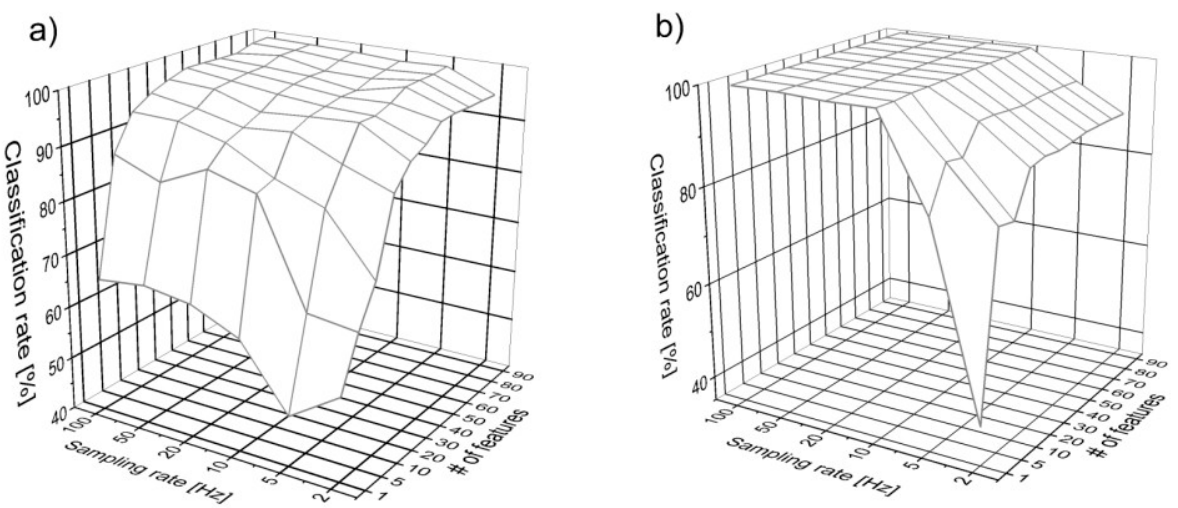

Figure 3. Classification rate of the hydraulic system depending on sampling rate (w/o AA) and number of features used: (a) accumulator with constant working cycle, (b) valve with randomized working cycle determined by 10 -fold cross-validation with Mahalanobis distance classifier. 
Table 1. Classification rate of remaining useful lifetime of the EMC, if the data analysis system is allowed to select the best feature extraction method based on 10-fold cross-validation. BFC: Best Fourier Coefficients; PCA: Principal Component Analysis.

\begin{tabular}{cccc}
\hline Sampling Rate & $\begin{array}{c}\text { Maximum Classification } \\
\text { Rate }\end{array}$ & $\begin{array}{c}\text { Feature Extraction } \\
\text { Method }\end{array}$ & $\begin{array}{c}\text { Number of } \\
\text { Features }\end{array}$ \\
\hline $2000 \mathrm{~Hz}$ & $95 \%$ & BFC & 496 \\
$1000 \mathrm{~Hz}$ & $94 \%$ & BFC & 493 \\
$500 \mathrm{~Hz}$ & $91 \%$ & PCA & 197 \\
$100 \mathrm{~Hz}$ & $90 \%$ & PCA & 221 \\
$50 \mathrm{~Hz}$ & $85 \%$ & PCA & 319 \\
\hline
\end{tabular}
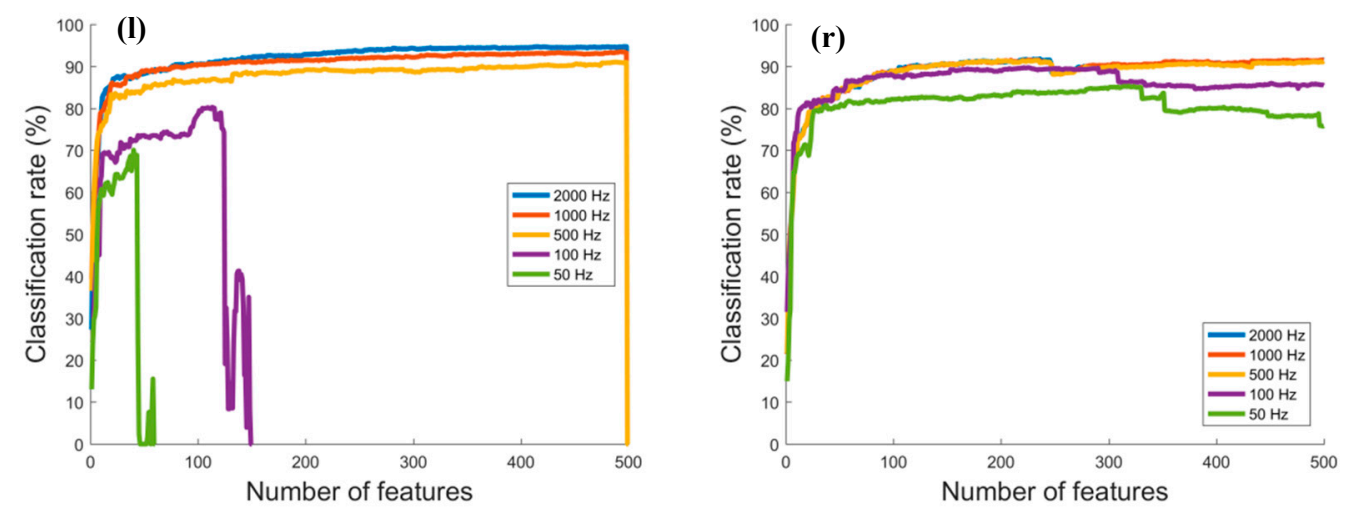

Figure 4. Classification rate of remaining useful lifetime estimation of EMC depending on sampling rate (with AA) and number of features used. Left (l): features extracted from frequency domain using best Fourier coefficients; Right (r): features extracted from overall cycle shape using PCA. The classification rate was determined by 10-fold cross-validation with Mahalanobis distance classifier. PCA feature extraction outperforms BFC for low sampling rates as this is more affected by reduced sample rates.

\section{Discussion}

The analysis based on these examples helps to configure the data acquisition of sensor networks for multivariate condition monitoring more efficiently with a minimum data rate, thus drastically reducing the hardware requirements for signal acquisition as well as processing and therefore improves the feasibility of novel condition monitoring in industrial environments. However, it is unclear how to determine the lowest sampling rate that allows both reliable fault classification (required classification rate depends on application) and low hardware costs in advance. As a result an initial testbed with a sampling rate that is sufficiently high to capture all expected fault symptoms like high frequency harmonics is required to generate a dataset that can be used for successive resampling to approximate the minimum sampling rate needed for the desired classification rate.

Author Contributions: The data analysis and writing has been done by N.H. (hydraulic machine) and T.S. (EMC). EMC experiments were conceived and performed by N.H. and S.K. A.S. is project leader of iCM Hydraulics (hydraulic machine) and MoSeS-Pro (EMC experiments) and scientific supervisor.

Funding: MoSeS-Pro: German Federal Ministry of Education and Research, funding code 16ES0419K; iCM Hydraulics: EFI program (support of development, research, and innovation in Saarland), research by ZeMA was financed by HYDAC Filter Systems.

Conflicts of Interest: The authors declare no conflict of interest. The founding sponsors had no role in the design of the study; in the collection, analyses, or interpretation of data; in the writing of the manuscript, and in the decision to publish the results. 


\section{References}

1. Helwig, N.; Pignanelli, E.; Schütze, A. Condition monitoring of a complex hydraulic system using multivariate statistics. In Proceedings of the IEEE Instrumentation and Measurement Technology Conference (I2MTC), Pisa, Italy, 11-14 May 2015.

2. Helwig, N.; Schneider, T.; Schütze, A. Modular Sensor Systems for real time Process Control and Smart Condition Monitoring using XMR sensor technology. In Proceedings of 14th Symposium Magnetoresistive Sensors and Magnetic Systems, Wetzlar, Germany, 21-22 March 2017.

3. Duda, R.O.; Hart, P.E.; Stork, D.G. Pattern Classiflcation, 2nd ed.; Wiley: New York, NY, USA, 2000.

4. Schneider, T.; Helwig, N.; Schütze, A. Automatic feature extraction and selection for classification of cyclical time series data. tm-Tech. Mess. 2017, 84, 198-206.

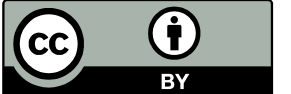

(C) 2018 by the authors. Licensee MDPI, Basel, Switzerland. This article is an open access article distributed under the terms and conditions of the Creative Commons Attribution (CC BY) license (http://creativecommons.org/licenses/by/4.0/). 